\title{
ALMOST PERIODICITY, EQUI-CONTINUITY AND TOTAL BOUNDEDNESS
}

\author{
W. H. GOTTSCHALK
}

Let $X$ be a uniform space; that is to say, let $X$ be a space provided with a system of indexed neighborhoods $U_{\alpha}(x)(x \in X, \alpha=$ index), subject to the conditions (A. Weil): (1) If $x \in X$ and if $\alpha$ is an index, then $x \in U_{\alpha}(x)$; (2) If $\alpha$ and $\beta$ are indices, then there exists an index $\gamma$ such that $x \in X$ implies $U_{\gamma}(x) \subset U_{\alpha}(x) \cap U_{\beta}(x)$; (3) If $\alpha$ is an index, then there exists an index $\beta$ such that $x, y, z \in X$ with $x, y \in U_{\beta}(z)$ implies $x \in U_{\alpha}(y)$. Let $T$ be a topological group with identity $\sigma$ and let $f$ be a transformation of $X \times T$ into $X$. We agree to write $f^{t}(x)$ or $f_{x}(t)$ in place of $f(x, t) \quad(x \in X, t \in T)$, whenever we wish. Furthermore, let $f$ define a transformation group; that is to say, suppose $f^{\sigma}(x)=x$ and $f^{s} f^{t}(x)=f^{t s}(x) \quad(x \in X ; t, s \in T)$. We impose continuity conditions on $f$ as the needs arise.

A subset $E$ of $T$ is said to be relatively dense provided there exists a compact set $A$ in $T$ such that each left translate of $A$ intersects $E$. A point $x$ of $X$ is called almost periodic provided that if $U$ is a neighborhood of $x$, then there exists a relatively dense set $E$ in $T$ for which $f(x, E) \subset U$. We observe that the notion of almost periodic point depends on the topology in $T$, the strongest type of almost periodicity occurring when $T$ is provided with the discrete topology. It is easily proved that a set $E$ in $T$ is relatively dense if and only if there exists a compact set $B$ in $T$ such that $T=E B$. The set $f(x, T)$ is called the orbit of the point $x$.

TheOREM 1. If the family $\left[f^{t} \mid t \in T\right]$ is equi-continuous at $x$, if $f_{x}$ is continuous on $T$, and if $x$ is almost periodic, then the orbit of $x$ is totally bounded. Conversely, if the family $\left[f^{t} \mid t \in T\right]$ is equi-uniformly continuous and if the orbit of $x$ is totally bounded, then $x$ is almost periodic.

Proof. Suppose the hypotheses of the first statement hold. Let $\alpha$ be an index. There exists an index $\beta$ such that the $\beta$-neighborhood of each compact set in $X$ is contained in the union of finitely many $\alpha$-neighborhoods. By hypothesis we can find an index $\gamma$ such that $f^{t}\left(U_{\gamma}(x)\right) \subset U_{\beta}\left(f^{t}(x)\right)(t \in T)$. There are sets $E$ and $A$ in $T$ such that $T=E A, A$ is compact, and $f(x, E) \subset U_{\gamma}(x)$. Hence, $f(x, T) \subset f\left(U_{\gamma}(x), A\right)$ $\subset U_{\beta}(f(x, A))$. Since $f(x, A)$ is compact, $U_{\beta}(f(x, A))$ is contained in

Presented to the Society, April 27, 1946; received by the editors March 11, 1946. 
the union of finitely many $\alpha$-neighborhoods. Thus the orbit of $x$ is totally bounded.

Suppose the hypotheses of the second statement hold. Let $\alpha$ be an index. By hypothesis there exists an index $\beta$ such that $f^{t}\left(U_{\beta}(y)\right)$ $\subset U_{\alpha}\left(f^{t}(y)\right)(y \in X, t \in T)$. Choose finitely many elements $t_{1}, \cdots, t_{n}$ of $T$ so that $f(x, T) \subset \bigcup_{i=1}^{n} V_{i}$, where $V_{i}=U_{\beta}\left(f\left(x, t_{i}\right)\right)$. If $t \in T$, then for some $i, f(x, t) \in V_{i}$ whence $f\left(x, t t_{i}^{-1}\right) \in U_{\alpha}(x)$. Hence, $x$ is almost periodic.

CoRollary 1. If the family [ $\left.f^{t} \mid t \in T\right]$ is equi-uniformly continuous, if $f_{x}$ is continuous on $T$, and if $x$ is almost periodic, then $x$ is almost periodic with respect to the discrete topology in $T$.

Corollary 2 (Bochner's Theorem). If $x(\tau),-\infty<\tau<+\infty$, is $a$ complex-valued continuous function, then $x(\tau)$ is an almost periodic function in the sense of Bohr if and only if each sequence of translates of $x(\tau)$ contains a uniformly convergent subsequence.

Proof. Define $Y$ to be the collection of all complex-valued continuous functions $y(\tau),-\infty<\tau<+\infty$, and define indexed neighborhoods in $Y$ so that $y \in U_{n}\left(y_{0}\right)$ if and only if $\left|y(\tau)-y_{0}(\tau)\right|<1 / n$ $(-\infty<\tau<+\infty)$ where the index $n$ is a positive integer. Construct a transformation group $g$ in $Y$ by translation of the functions in $Y$, taking $T$ to be the additive group of reals with its natural topology. Now $x$ is an almost periodic point if and only if $x(\tau)$ is an almost periodic function. Clearly, the family $\left[g^{t} \mid t \in T\right]$ is equi-uniformly continuous. Also if $x(\tau)$ is an almost periodic function, then $x(\tau)$ is uniformly continuous whence $g_{x}$ is continuous on $T$. Hence, by Theorem $1, x$ is an almost periodic point if and only if the orbit of $x$ is totally bounded. The conclusion follows.

We point out that A. Weil [2, pp. 130-133 $]^{1}$ has essentially taken Theorem 1 as the definition of an almost periodic point with respect to a transformation group.

If $X$ is an arbitrary set, if $Y$ is a uniform space, and if $\Phi$ is a nonvacuous collection of functions on $X$ to $Y$, then we consider $\Phi$ to be a uniform space in the following manner: If $\alpha$ is an index belonging to $Y$ and if $\phi \in \Phi$, then the $\alpha$-neighborhood $U_{\alpha}(\phi)$ of $\phi$ is taken to be the set of all elements $\psi$ of $\Phi$ such that $\psi(x) \in U_{\alpha}(\phi(x))$ for every element $x$ of $X$.

The following lemma will be recognized as a generalization of Ascoli's theorem and its converse.

Lemma 1. Let $X$ and $Y$ be uniform spaces and let $\Phi$ be a nonvacuous

${ }^{1}$ Numbers in brackets refer to the Bibliography at the end of the paper. 
collection of functions on $X$ to $Y$. If $X$ and $Y$ are totally bounded and if the family $\Phi$ is equi-uniformly continuous, then the space $\Phi$ is totally bounded. Conversely, if the individual functions in $\Phi$ are uniformly continuous and if the space $\Phi$ is totally bounded, then the family $\Phi$ is equiuniformly continuous.

Proof. Suppose $X$ and $Y$ are totally bounded and $\Phi$ is equi-uniformly continuous. Let $\alpha$ be an index belonging to $\Phi$ and, hence, to $Y$. Choose an index $\beta$ belonging to $Y$ so that $a, b, c \in Y$ with $a, b \in U_{\beta}(c)$ implies $a \in U_{\alpha}(b)$. We can find an index $\gamma$ belonging to $Y$ and finitely many points $y_{1}, \cdots, y_{n}$ of $Y$ so that $y \in Y$ implies $U_{\gamma}(y) \subset U_{\beta}\left(y_{j}\right)$ for some integer $j, 1 \leqq j \leqq n$. There exists an index $\delta$ belonging to $X$ such that $\phi\left(U_{\delta}(x)\right) \subset U_{\gamma}(\phi(x)),(\phi \in \Phi, x \in X)$. Choose finitely many points $x_{1}, \cdots, x_{m}$ of $X$ so that $X=\bigcup_{i=1}^{m} U_{\delta}\left(x_{i}\right)$. Hence if $\phi \in \Phi$, then to each integer $i, 1 \leqq i \leqq m$, there corresponds at least one integer $j, 1 \leqq j \leqq n$, such that $\phi\left(U_{\delta}\left(x_{i}\right)\right) \subset U_{\beta}\left(y_{j}\right)$. Letting $I, J$ denote the first $m, n$ positive integers, each element $\phi$ of $\Phi$ defines in the obvious manner a class $C(\phi)$ of transformations of $I$ into $J$. Choose finitely many elements $\phi_{1}, \cdots, \phi_{r}$ of $\Phi$ so that the class $U_{k=1}^{r} C\left(\phi_{k}\right)$ is maximal. It follows that $\Phi=\cup_{k=1}^{r} U_{\alpha}\left(\phi_{k}\right)$. Hence, $\Phi$ is totally bounded.

Suppose now that the functions in $\Phi$ are uniformly continuous and $\Phi$ is totally bounded. Let $\alpha$ be an index belonging to $Y$. There exists an index $\gamma$ belonging to $Y$ so that $a \in U_{\gamma}(c), b \in U_{\gamma}(d)$ and $c \in U_{\gamma}(d)$ implies $a \in U_{\alpha}(b)$. Choose finitely many elements $\phi_{1}, \cdots, \phi_{n}$ of $\Phi$ so that $\Phi=\bigcup_{i=1}^{n} U_{\gamma}\left(\phi_{i}\right)$. Select indices $\beta(i)(i=1, \cdots, n)$ belonging to $X$ which have the property that $\phi_{i}\left(U_{\beta(i)}(x)\right) \subset U_{\gamma}\left(\phi_{i}(x)\right)(x \in X$; $i=1, \cdots, n)$. There exists an index $\beta$ belonging to $X$ for which $U_{\beta}(x) \subset \bigcap_{i=1}^{n} U_{\beta(i)}(x)(x \in X)$. We conclude that $\phi\left(U_{\beta}(x)\right) \subset U_{\alpha}(\phi(x))$ $(\phi \in \Phi, x \in X)$. Hence, $\Phi$ is equi-uniformly continuous and the proof is completed.

We say that the transformation group $f$ is almost periodic provided that if $\alpha$ is an index, then there exists a relatively dense set $E$ in $T$ for which $x \in X$ implies $f(x, E) \subset U_{\alpha}(x)$. It may be verified that in order for $f$ to be almost periodic it is both necessary and sufficient that if $\alpha$ is an index, then there exists a compact set $A$ in $T$ such that to each element $t$ in $T$ there corresponds an element $s$ in $A$ for which $f^{t}(x) \in U_{\alpha}\left(f^{s}(x)\right)(x \in X)$. If $T$ has the discrete topology, this characterization reduces essentially to Montgomery's definition [1, p. 323] of an almost periodic transformation group.

In the following theorem we denote $\left[f^{t} \mid t \in T\right]$ by $G$ and, somewhat improperly, call $G$ the transformation group.

Theorem 2. If $X$ is compact and if $f$ is continuous on $X \times T$, then 
the following statements are pairwise equivalent: (1) The transformation group $G$ is almost periodic; (2) The family $G$ is equi-uniformly continuous; (3) The space $G$ is totally bounded.

Proof. By Lemma 1, (2) is equivalent to (3).

Suppose (1) is satisfied. Let $\alpha$ be an index. There exists an index $\gamma$ such that $a \in U_{\gamma}(c), b \in U_{\gamma}(d)$ and $c \in U_{\gamma}(d)$ implies $a \in U_{\alpha}(b)$. It is possible to find a compact set $A$ in $T$ such that if $t \in T$, then $f^{t}(x) \in U_{\gamma}\left(f^{s}(x)\right)(x \in X)$, for some element $s$ in $A$. Since $f$ is uniformly continuous on $X \times A$, we can choose an index $\beta$ for which $f^{s}\left(U_{\beta}(x)\right)$ $\subset U_{\gamma}\left(f^{s}(x)\right) \quad(x \in X, s \in A)$. It follows that $f^{t}\left(U_{\beta}(x)\right) \subset U_{\alpha}\left(f^{t}(x)\right)$ $(x \in X, t \in T)$. Hence, (2) is satisfied.

Suppose (2) is satisfied. Let $\alpha$ be an index. There exists an index $\beta$ such that $f^{t}\left(U_{\beta}(x)\right) \subset U_{\alpha}\left(f^{t}(x)\right) \quad(x \in X, t \in T)$. Since $G$ is totally bounded, we can select finitely many elements $t_{1}, \cdots, t_{n}$ in $T$ so that $G=\cup_{i=1}^{n} U_{\beta}\left(f^{t i}\right)$. Let $t$ be an element of $T$. Then for some $i$, $f(x, t) \in U_{\beta}\left(f\left(x, t_{i}\right)\right) \quad(x \in X)$, whence $f\left(x, t t_{i}^{-1}\right) \in U_{\alpha}(x) \quad(x \in X)$. Thus (1) is satisfied and the proof is completed.

CoRollary 3. If $X$ is compact, if $f$ is continuous on $X \times T$, and if $f$ is almost periodic, then $f$ is almost periodic with respect to the discrete topology in $T$.

Corollary 4 (Sharpened Dirichlet-Kronecker Theorem). If $t, a_{1}, \cdots, a_{k}$ are nonzero real numbers and if $\epsilon$ is a positive number, then there exists a relatively dense set $N$ of integers such that $n \in N$ implies the existence of integers $m_{1}, \cdots, m_{k}$ for which $\left|n t-m_{i} a_{i}\right|<\epsilon$ $(i=1, \cdots, k)$.

Proof. Let $X_{1}, \cdots, X_{k}$ denote pairwise disjoint circle boundaries in the plane with circumferences $\left|a_{1}\right|, \cdots,\left|a_{k}\right|$. Take $X=\bigcup_{i=1}^{k} X_{i}$ with the natural uniformity. Define the uniformity-preserving homeomorphism $\phi$ of $X$ onto $X$ by rotating each circle $X_{i}$ through arc length $t$. The transformation group generated by the integral powers of $\phi$ satisfies (2) of Theorem 2 and, hence, is almost periodic. The conclusion follows from the definition of almost periodicity.

\section{BIBLIOGRAPHY}

1. Deane Montgomery, Almost periodic transformation groups, Trans. Amer. Math. Soc. vol. 42 (1937) pp. 322-332.

2. A. Weil, L'intégration dans les groupes topologiques et ses applications, Actualités Scientifiques et Industrielles, Paris, Hermann, 1938.

University of Pennsylvania 